\title{
Revista Brasileira de Enfermagem REBEn \\ Manejo da dor pós-operatória na Enfermagem Pediátrica: em busca de subsídios para aprimorar o cuidado
}

\author{
Managing postoperative pain in Pediatric Nursing: searching for subsides \\ to improve nursing care \\ El manejo del dolor postoperatorio en la Enfermería Pediátrica: en búsqueda de \\ apoyo para mejorar el cuidado
}

\section{Fernanda Cristina Queiroz}

Enfermeira do Hospital das Clínicas de Ribeirão Preto da Universidade de São Paulo, Ribeirão Preto, SP.

Especialista em Enfermagem em Cuidado Intensivo Neonatal e Pediátrico, Mestranda do Programa de Enfermagem em Saúde Pública do Departamento de Enfermagem MaternoInfantil e Saúde Pública da EERP-USP. fcq_enf@yahoo.com.br

\section{Lucila Castanheira Nascimento}

Adriana Moraes Leite

Milena Flória-Santos

Regina Aparecida Garcia de Lima

Carmen Gracinda Silvan Scochi

Docentes do Departamento de Enfermagem Materno-Infantil e Saúde Pública da EERP. USP, Ribeirão Preto, SP.

Projeto inserido no Grupo de Estudos em Saúde da Criança e do Adolescente. Escola de Enfermagem de Ribeirão Preto da Universidade de São Paulo (EERP. USP), Centro Colaborador da OMS para o Desenvolvimento da Pesquisa em Enfermagem. Departamento de Enfermagem Materno-Infantil e Saúde Pública. E-mail: lucila@eerp.usp.br

\section{RESUMO}

O objetivo deste artigo é realizar uma revisão da literatura sobre o manejo da dor pelos profissionais de enfermagem no pós-operatório infantil, no período de 1993 a 2005. A revisão possibilitou identificar três temáticas: fatores que influenciam o manejo da dor da criança pelos enfermeiros, intervenções para 0 alívio da dor da criança e avaliação e resposta dos enfermeiros à experiência de dor da criança. 0 manejo da dor infantil é um ato complexo que engloba elementos das dimensões referentes à própria criança, aos profissionais de saúde e aos seus familiares. A carência de estudos nesta área revela a necessidade de se realizarem pesquisas, para que se possa (re)pensar o cuidado de enfermagem pediátrica.

Descritores: Dor; Criança; Enfermagem; Dor pós-operatória.

\section{ABSTRACT}

This study reports on a literature review about child postoperative pain management by nursing professionals, in the period from 1993 to 2005. Three themes were identified: factors influencing nurses' management of child pain, interventions to relieve child pain, and nurses' assessment and response to children's pain experience. Child pain management is a complex act that involves elements of the dimensions related to the children themselves, health professionals and family members. The lack of studies in this area reveals the need for research, with a view to (re)considering pediatric nursing care.

Descriptors: Pain; Child; Nursing; Pain, postoperative.

\section{RESUMEN}

La finalidad de este artículo es realizar una revisión de la literatura sobre el manejo del dolor por los profesionales de enfermería en el postoperatorio infantil, en el período de 1993 a 2005. La revisión posibilitó identificar tres temáticas: factores que influencian el manejo del dolor del niño por los enfermeros, intervenciones para el alivio del dolor del niño y evaluación y respuesta de los enfermeros a la experiencia de dolor del niño. El manejo del dolor infantil es un acto complejo que abarca elementos de las dimensiones referentes al propio niño, a los profesionales de salud y a sus familiares. La falta de estudios en esta área revela la necesidad de llevar a cabo investigaciones, para que se pueda (re)pensar el cuidado de enfermería pediátrica.

Descriptores: Dolor; Niño; Enfermería; Dolor postoperatorio.

Queiroz FC, Nascimento LC, Leite AM, Flósria-Santos M, Lima RAG, Scochi CGS. Manejo da dor pósoperatória na Enfermagem Pediátrica: em busca de subsídios para aprimorar o cuidado. Rev Bras Enferm 2007 jan-fev; 60(1):87-91.

\section{INTRODUÇÃO}

A dor é uma experiência que se caracteriza pela complexidade, subjetividade e multidimensionalidade ${ }^{(1)}$. Deve ser vista como uma experiência complexa que envolve o organismo como um todo e não somente os componentes fisiológicos. Dessa forma, compreende os aspectos psicológicos e sociais da vida do indivíduo, não podendo ser reduzida ao sofrimento físico(1).

Embora muitos pesquisadores tenham como objeto de estudo a preocupação com a existência da dor em crianças, bem como sua medida, o tema ainda é muito pouco explorado e, muitas vezes, subestimado por profissionais de saúde que convivem freqüentemente com a presença da dor na criança(2). O manejo da dor pelos profissionais de saúde para essa população é freqüentemente inadequado ${ }^{(3,4)}$ e tem sido bem documentado que um número expressivo de crianças recebe doses subterapêuticas de analgésicos, ocasionando um subtratamento da dor destas crianças ${ }^{(5-8)}$. $O$ conhecimento sobre a dor e seus métodos de alívio, experiências profissionais e pessoais sobre a dor, crenças e atitudes dos enfermeiros são alguns fatores que se relacionam à submedicação e ao subtratamento da dor ${ }^{(3,7,9-11)}$. Além disso, tem sido 
documentada a discrepância no tipo e quantidade de analgésicos prescritose administrados em crianças e adultos que passam por cirurgias similares, sendo observado que, às crianças, são administrados menos analgésicos ${ }^{(8,9,12-14)}$.

Mesmo com o aumento da atenção dada às intervenções para o alívio da dor pediátrica nas últimas décadas, ainda hoje, crianças são submetidas a uma gama de procedimentos dolorosos sem sedação e analgesia adequadas e sem atenção ao conforto do doente ${ }^{(12)}$. No ambiente hospitalar, a cirurgia é uma das maiores causas de dor aguda, assim, a dor pós-operatória é representativa para o estudo da dor aguda que se diferencia das outras dores, crônica e maligna, por trazer consigo a expectativa de alívio e resolução num espaço de tempo ${ }^{(15)}$

A dor pós-operatória continua sendo tratada inadequadamente, e pacientes ainda sofrem moderada ou severa dor após a cirurgia. Crianças submetidas a cirurgias experimentam dor no período pós-operatório, e o primeiro e mais importante objetivo da analgesia pós-operatória é 0 alívio dessa dor ${ }^{\left({ }^{16}\right)}$. $A$ analgesia visa também a maximizar o resultado da cirurgia e minimizar 0 traumatismo da anestesia, durante o período de recuperação. A dor pósoperatória facilita o surgimento de complicações respiratórias, como atelectasias, pneumonias e hipóxia, de complicações gastrointestinais, ocasionadas pela imobilização, pela trombose venosa e supressão do sistema imune; 0 alívio dessa dor reduz essas complicações ${ }^{(11,12,16,17)}$.

Frente à necessidade de ampliar o conhecimento a respeito da temática, o objetivo deste artigo é realizar uma revisão da literatura de pesquisas que abordem o manejo da dor pelos profissionais de enfermagem no pósoperatório, no período de 1993 a 2005, a fim de identificar os temas de maior interesse e fornecer subsídios para um cuidado de enfermagem de maior qualidade às crianças com dor.

\section{METODOLOGIA}

Trata-se de uma revisão bibliográfica, realizada a partir das bases de dados LILACS, MEDLINE e CINAHL e em artigos disponibilizados on-line, no período de 1993 a 2005. Fez-se também uma busca não sistematizada nos principais periódicos da área e por meio de referências bibliográficas existentes nos próprios artigos selecionados e encontrados. Foram utilizadas as seguintes palavras-chave, em diferentes combinações: pain, child, nursing e pain, postoperative, sendo identificados, 16 artigos pertinentes aos objetivos do estudo.

A partir da leitura dos resumos, os artigos foram selecionados tendo em vista critérios de inclusão e exclusão. Foram incluídos artigos publicados em periódicos nacionais e internacionais, redigidos nas línguas inglesa, portuguesa e espanhola, no período de 1993 a 2005, independente da abordagem metodológica. Incluímos artigos relacionados ao manejo da dor da criança no pós-operatório e que tinham como participantes enfermeiras ou equipe de enfermagem, mesmo que outros sujeitos tivessem sido objetos de estudo, como as próprias crianças e seus pais. Excluíram-se as publicações que não apresentavam resumo que enfocavam validação de escalas, dosagens de medicamentos e procedimentos cirúrgicos. Com base nos critérios apresentados, a amostra deste estudo constituiu-se de 11 artigos.

Para análise dos artigos, utilizamos um roteiro contendo os seguintes indicadores: ano, temática central, participantes, abordagem metodológica e resultado principal.

\section{RESULTADOS}

Dos 11 artigos analisados, dez foram desenvolvidos por pesquisadores da área de enfermagem e um deles por um farmacêutico. Quanto ao ano de publicação, houve uma homogeneidade na distribuição dos artigos, porém os anos de 1999 e 2001 foram os anos em que mais se produziu sobre 0 manejo da dor pós-operatória em crianças pelos profissionais de enfermagem.

Em relação ao país de publicação, não houve nenhum artigo publicado no Brasil. Os artigos resultaram de produções de pesquisadores provenientes dos seguintes países: Canadá, Reino Unido, Islândia, Dinamarca, Holanda, Estados Unidos e Finlândia, sendo que esses dois últimos foram os que mais produziram, com quatro e três artigos, respectivamente.

Para caracterizar a metodologia, foram utilizadas as denominações trazidas pelos próprios autores. Assim, dos 11 artigos analisados, sete eram quantitativos, três qualitativos (sendo dois estudos fenomenológicos e um que utilizou a teoria fundamentada em dados) e uma revisão de literatura.

A análise das publicações selecionadas permitiu a identificação de três temáticas: fatores que influenciam o manejo da dor da criança pelos enfermeiros, intervenções para o alívio da dor da criança e avaliação e resposta dos enfermeiros à experiência de dor da criança.

\section{Fatores que influenciam o manejo da dor da criança pelos enfermeiros}

Por meio de um estudo fenomenológico, realizaram-se entrevistas semiestruturadas com 20 enfermeiras e 20 pais, a fim de examinar a comunicação entre estes a respeito da dor infantil e como isso poderia ajudar no manejo da dor pós-operatória em crianças ${ }^{(10)}$. Como resultado viu-se que há uma comunicação precária, entre os pais e as enfermeiras, que pode ser explicada pela falta de conhecimento destas últimas, e que realimenta a falta de comunicação com os pais para informar sobre o manejo da dor no pósoperatório. Este estudo contribui com reflexões sobre quando e como as informações devem ser dadas, tendo em vista a individualidade do cuidado.

Propondo-se a examinar as relações entre o conhecimento das enfermeiras e suas atitudes no alívio da dor de crianças, além das habilidades para superar barreiras para um melhor manejo da dor, desenvolveu-se um estudo quantitativo aplicando-se um questionário a 67 enfermeiras e 132 crianças $^{(9)}$. Para mensurar o conhecimento e as atitudes das enfermeiras, aplicou-se um instrumento com 33 itens que incluiam questões sobre o uso, as ações e os efeitos dos analgésicos, intervenções não-farmacológicas para o alívio da dor, avaliação da dor, lista de analgésicos e a segurança dos opióides para as crianças. Para mensurar a dor das crianças, utilizou-se uma escala fotográfica, contendo seis fotos de faces de crianças representando as fases de dor. $O$ estudo evidenciou que as enfermeiras medicavam as crianças quando estas sentiam dor, além de mostrar que enfermeiras com maior conhecimento e atitudes mais positivas possuíam maior habilidade de transpor barreiras para um melhor manejo da dor infantil.

Para descrever os fatores que promovem e retardam o uso de métodos não-farmacológicos pelas enfermeiras para aliviar a dor cirúrgica de crianças, conduziu-se um estudo quantitativo, por meio da aplicação de um questionário, tipo Likert, a 162 enfermeiras ${ }^{(3)}$. Como fatores que promovem o uso dos métodos não-farmacológicos estão as habilidades das enfermeiras, a diversidade dos métodos de alívio da dor, o excesso de trabalho, a idade e a habilidade de cooperação da criança e a participação dos pais. Entre os fatores que impedem o uso desses métodos estão a insegurança das enfermeiras, a habilidade da criança em expressar dor, as limitações dos métodos de alívio da dor e o processo de trabalho. Além destes resultados, o estudo contribui com dados que mostram também que enfermeiras mais velhas e com maior experiência no trabalho são mais competentes e menos inseguras na utilização de métodos não-farmacológicos, quando comparadas às enfermeiras mais jovens e com menor experiência.

Um estudo quantitativo buscou avaliar o resultado da implementação de um programa de capacitação em serviço sobre a dor pediátrica na prática de enfermagem ${ }^{(18)}$. O programa incluiu o relato histórico e pesquisas sobre 0 manejo da dor pediátrica, mitos e concepções errôneas, avaliação das escalas de mensuração da dor, intervenções farmacológicas e não-farmacológicas, benefícios do alívio da dor, considerações éticas e envolvimento dos pais. Aplicou-se um questionário de múltipla escolha a 52 enfermeiras. Este programa, de participação obrigatória, teve duração de três horas e, ao final do período, foram feitos estudos clínicos, intervenções para o tratamento da dor e auditoria nos prontuários. Ao contrário do que era esperado, após a implementação do programa, houve um aumento do tempo de administração 
de analgésico antes da primeira dose e entre as doses de analgésicos dadas aos pacientes. As autoras sugerem explicações para este resultado: talvez as enfermeiras tivessem incorporado em sua prática o uso de métodos nãofarmacológicos para o alívio da dor aprendidos durante o programa ou talvez elas acreditassem que já tinham conhecimento suficiente sobre a dor e não necessitavam de ampliar seu conhecimento, ou ainda, que a mudança na prática profissional das enfermeiras, após a participação no programa, pode ter sido interpretada como uma crítica ao cuidado que elas prestavam às crianças.

\section{Intervenções para o alívio da dor da criança}

Uma revisão de literatura foi realizada na intenção de se identificar a presença de pesquisas que mostram se crianças recebem medicação insuficiente para dor no pós-operatório, os fatores que explicam esta inadequada administração de analgésicos e quais intervenções são necessárias para o alívio da dor ${ }^{(19)}$. Foram analisados nove estudos e os resultados mostraram que a criança é submedicada, sendo que o alívio de sua dor é insuficiente devido à falta de conhecimento. Além disso, ao comparar a prescrição e administração de analgésicos entre crianças e adultos, os últimos são mais medicados.

Para descrever o uso de técnicas não-farmacológicas pelas enfermeiras, para o alívio da dor pós-operatória em crianças, um estudo quantitativo foi feito enviando questionário a 162 enfermeiras ${ }^{(20)}$. A análise mostrou que muitos métodos não-farmacológicos são usados para o alívio da dor pósoperatória, tais como: o suporte emocional, auxiliar nas atividades diárias e criar um ambiente confortável são usados rotineiramente; o comportamento cognitivo e os métodos físicos são utilizados com menor freqüência. Contudo, as autoras alertam que maior atenção deve ser dada a outros tipos de estratégias, como o uso de imagem, relaxamento e massagem.

Buscando descrever a percepção das enfermeiras sobre um guia para os pais para o alívio da dor cirúrgica da criança, conduziu-se um estudo quantitativo, enviando questionário a 162 enfermeiras ${ }^{(21)}$. Como resultado, as enfermeiras necessitam dar mais informação preparatória aos pais e informálos sobre o seu papel no cuidado de suas crianças, como o significado do contato entre pais e filhos e a possibilidade de estar presentes e fazer parte do cuidado no pós-operatório de suas crianças. Com relação aos métodos nãofarmacológicos no cuidado das crianças, os mais freqüentemente ensinados são o suporte emocional, a ajuda nas atividades diárias e o posicionamento; entretanto, a pesquisadora reporta que maior atenção deve ser dada a estratégias como imagem, reforço positivo, regulação térmica e massagem na prática de enfermagem.

\section{Avaliação e resposta dos enfermeiros à experiência de dor das crianças}

Um estudo qualitativo, usando a teoria fundamentada em dados, foi conduzido para descrever como os pais e as enfermeiras respondem à experiência de dor da criança hospitalizada após intervenções cirúrgicas ${ }^{(14)}$. Realizaram-se entrevistas com 11 crianças, 22 pais e 24 enfermeiras, além da observação que buscava identificar comportamentos de cuidados e 0 contexto do cuidado de cada criança que experienciava dor pós-operatória. Os achados mostraram que as enfermeiras priorizavam o cuidado técnico, apresentavam limitada avaliação da dor e não eram capazes de aliviar adequadamente a dor das crianças. Os pais promoviam cuidados por meio do conforto e da monitorização vigilante da dor das crianças. Nenhum resultado foi apresentado em relação às entrevistas com as crianças.

Buscando explorar a percepção de pais e enfermeiras em relação ao envolvimento e manejo da dor das crianças e os possíveis efeitos do envolvimento dos pais no manejo dessa dor, um estudo fenomenológico foi feito, no qual 20 pais e 20 enfermeiras foram entrevistados ${ }^{(22)}$. 0 envolvimento dos pais no manejo da dor de suas crianças é superficial e limitado. Eles relatam ter um papel passivo em relação ao cuidado com a dor de seus filhos, levando a um sentimento de frustração. As enfermeiras relatam que há um adequado envolvimento dos pais e um adequado manejo da dor das crianças. $\mathrm{O}$ autor desse estudo concluiu que, pelo fato das respostas dos participantes serem opostas, isto poderia demonstrar que há uma lacuna na comunicação entre pais e enfermeiras.

Com o objetivo de examinar a relação entre a avaliação da dor realizada na perspectiva das crianças e das enfermeiras, na intenção de identificar se estas avaliações são discrepantes ou não, um estudo quantitativo foi feito com 100 crianças e duas enfermeiras ${ }^{(23)}$. Para avaliação da dor, as crianças utilizaram a escala poker chip tool e as enfermeiras a escala analógica visual. Como resultado, as enfermeiras subestimam a dor das crianças e não sabem interpretar essa dor.

Fez-se um estudo quantitativo aplicando-se um questionário a 260 enfermeiras, com o objetivo de descrever a percepção destas sobre sua prática na avaliação e manejo da dor pediátrica ${ }^{(8)}$. O estudo mostra que as enfermeiras usam a comunicação verbal, mudanças fisiológicas, expressão facial, comportamento não-verbal, mudanças nas atividades diárias, no estado neurológico, no afeto e na condição do paciente, como métodos para avaliar a dor. Para o manejo da dor, as enfermeiras utilizam métodos farmacológicos, como opióides e lidocaína, e métodos não-farmacológicos, como distração e relaxamento.

\section{DISCUSSÃO E IMPLICAÇÕES PARA A ENFERMAGEM}

Os dados analisados revelaram que a enfermagem é a categoria profissional que mais tem produzido sobre o manejo da dor pós-operatória em crianças, havendo apenas um artigo produzido por um farmacêutico. Com relação ao ano de publicação, percebe-se que houve uma preocupação maior com o tema em estudo a partir de 1999, evidenciando como é recente sua inserção em nosso meio.

Ao enfocarmos o país de publicação, o Brasil não apresentou nenhuma pesquisa sobre o tema, sendo que a maioria das publicações ficou com os Estados Unidos e a Finlândia. Isso mostra a necessidade de um maior conhecimento nesta área, a fim de compreender como os profissionais de enfermagem lidam com a dor pós-operatória em crianças, havendo a necessidade de compreender esta temática no nosso contexto, trazendo as especificidades culturais e socioeconômicas e comparando-as com os países que estão liderando as pesquisas nessa área. Além disso, o Brasil tem particularidades relacionadas ao cuidado de enfermagem, já que não são apenas as enfermeiras que o fazem e sim uma equipe de enfermagem constituída por outros elementos, como auxiliares e técnicos de enfermagem.

Em relação aos resultados dos estudos analisados, relacionados ao déficit na formação profissional dos enfermeiros para o manejo da dor em crianças, acreditamos que os mesmos podem ser transpostos à realidade brasileira, pois a abordagem deste tema foi recentemente incorporada na grade curricular do ensino superior. Neste sentido, o retorno desta formação na prática profissional daqueles recém-introduzidos no mercado de trabalho, bem como dos que já exercem suas atividades profissionais e necessitam de educação permanente para ampliação do conhecimento nesta área, demandará tempo e investimento para que se possa beneficiar de uma mudança na prática relacionada ao manejo da dor em criança. Neste sentido, destaca-se a política de Educação Permanente em Saúde do Sistema Único de Saúde, proposta e aprovada em 2003 , que pressupõe um trabalho articulado entre o sistema de saúde, em suas diversas esferas de gestão, e as instituições formadoras, com objetivo de contribuir para a transformação dos processos formativos e das práticas em saúde ${ }^{(24)}$. Outra possibilidade que facilitaria a introdução e discussão de conteúdos relacionados à temática da dor em crianças poderia ser por meio de atividades de extensão de serviços à comunidade, realizadas pela universidade, que contemplassem, por exemplo, métodos não-farmacológicos para o alívio da dor. Além disso, a condução de pesquisas nesta área, com a coleta de dados junto aos profissionais de enfermagem, pode contribuir para suscitar discussões e reflexões acerca do tema pela equipe como um todo. Os resultados inesperados apresentados no 
trabalho de Knoblauch e Wilson ${ }^{(18)}$ sugerem que o investimento apenas na educação permanente, com programas elaborados sem a participação dos profissionais, pode não ser a melhor escolha para se ampliar o conhecimento daqueles que atuam na enfermagem pediátrica.

A revisão possibilitou identificar os diversos fatores que influenciam o manejo da dor pós-operatória em crianças pelos enfermeiros. Os resultados das pesquisas analisadas identificaram que o manejo dessa dor é influenciado pelo déficit de conhecimento, pela comunicação inadequada entre enfermeiros e pais, pelas habilidades de transpor barreiras para um melhor manejo da dor, pela idade das enfermeiras, carga de trabalho, experiência profissional e pessoal sobre a dor, idade e gênero das crianças, bem como a habilidade delas para expressar a dor. Estes fatores nos alertam para a complexidade de elementos que interferem no manejo da dor pós-operatória de crianças, contribuindo para reflexões da equipe de enfermagem.

De acordo com 0 artigo de Pölkki et al(3), o excesso de trabalho não permite que os enfermeiros utilizem os métodos não-farmacológicos para aliviar a dor da criança. Este também é uma das dificuldades presentes na nossa realidade, pois grande parte dos profissionais de enfermagem possui jornada dupla de trabalho, o que os tornam cansados e desmotivados para implementar mudanças no cuidado à criança. Aliado a isso, este profissional tende a postergar a busca ou até mesmo excluir oportunidades de aprimoramento que lhes são oferecidas através de cursos, congressos e similares, que ampliem seu conhecimento e, conseqüentemente, contribuam para um cuidado de maior qualidade.

A equipe de enfermagem, por permanecer longos períodos com as crianças, encontra-se em vantagem para ensinar os pais sobre os aspectos que envolvem a dor pós-operatória de seus filhos e incluí-los no cuidado. Entretanto, estudos aqui analisados apontam que os enfermeiros, provavelmente a equipe de enfermagem no nosso contexto, possuem um déficit de conhecimento sobre 0 assunto, afastando-os dos pais, por se sentirem inseguros e ameaçados. Como conseqüência, estes pais podem afastar-se dos profissionais, temendo ser esta a atitude esperada por eles. Um ciclo vicioso se instala, com a prevalência de relações frouxas e comunicação descontínua, afetando a qualidade do cuidado. Imprime-se a necessidade de se reforçar a todos os profissionais que os familiares são nossos aliados, e não adversários, no cuidado com a criança com dor, pois eles são os que mais conhecem a criança e podem informar sobre qualquer mudança na sua condição, mesmo sendo a mais sutil.

O alívio da dor sempre foi parte essencial do cuidado das enfermeiras, mas estas não têm conseguido aliviá-la adequadamente por não reconhecêla, por não conseguir tratá-la e por aceitar que a dor severa faz parte da doença e do tratamento. Estas barreiras para o tratamento adequado da dor são ocasionadas pela falta de conhecimento sobre a sua avaliação e manejo(4).

Alguns estudos buscam compreender as intervenções para o alívio da dor pós-operatória da criança e, especificamente, o uso de métodos nãofarmacológicos pelas enfermeiras, como o suporte emocional, a ajuda nas atividades diárias e a criação de um ambiente confortável que foram os rotineiramente utilizados. Destaca-se a necessidade de se dar maior atenção a outros métodos, como 0 uso de imagem, massagem, relaxamento e reforço positivo na prática de enfermagem. Um estudo feito por Huth et al. ${ }^{(25)}$ mostrou que o uso da imagem, juntamente com a rotina analgésica, reduziu a dor e a ansiedade da criança após uma cirurgia ambulatorial e no domicílio. Especificamente na revisão aqui realizada, não foi encontrado nenhum estudo realizado no Brasil que tivesse como objeto de estudo a utilização de métodos não-farmacológicos para 0 alívio da dor pós-operatória em crianças, convidando a todos para esse desafio.

Com relação à avaliação e resposta do profissional de enfermagem frente à experiência de dor da criança, os artigos mostraram que as enfermeiras priorizam o cuidado técnico, têm limitada avaliação da dor e não são capazes de aliviar adequadamente a dor das crianças. Da mesma forma, pesquisas precisam ser conduzidas para que possamos investigar estas questões no nosso contexto.

O manejo da dor infantil é um ato de grande complexidade que engloba elementos das dimensões referentes à própria criança, aos profissionais de saúde e aos seus familiares, comumente representados pelos pais. Particularmente, o manejo da dor pós-operatória traz consigo outras particularidades e desafios que devem ser levados em consideração, não somente para a equipe de enfermagem, mas para todos os profissionais de saúde. Acarência de estudos que têm como foco a atuação dos profissionais de enfermagem no manejo da dor pós-operatória na criança, em nosso contexto, revela a necessidade de se realizarem pesquisas nesta área para que, por meio da adequada articulação entre pesquisa e prática, possamos (re)pensar o cuidado de enfermagem às crianças e inovar com práticas para o alívio da dor pós-operatória infantil.

\section{REFERÊNCIAS}

1. Gaiva MAM, Dias NS. Dor no recém-nascido: percepção de profissionais de saúde de um hospital universitário. Rev Paul Enferm 2002;21(3):234-9.

2. Claro MT. Escala de faces para avaliação da dor em crianças: etapa preliminar (dissertação). Ribeirão Preto (SP): Escola de Enfermagem de Ribeirão Preto, Universidade de São Paulo; 1993

3. Pölkki T, Laukkala H, Vehviläinen-Julkunen K, Pietilä AM. Factors influencing nurses' use nonpharmacological pain alleviation methods in paediatric patients. Scand J Caring Sci 2003;17:37383.

4. Manworren RCB. Pediatric nurses' knowledge and attitudes survey regarding pain. Pediatr Nurs 2000;26(6):610-4.

5. Cummings EA, Reid GJ, Finley A, McGrath PJ, Ritchie JA. Prevalence and source of pain in pediatric inpatients. Pain 1996;68:25-31.

6. American Academy of Pediatrics. American Pain Society. The assessment and management of acute pain in infants, children and adolescents. Pediatrics 2001;108: 793-7.

7. Salanterä S. Finnish nurses' attitudes to pain in children. J Adv Nurs 1999;29(3): 727-36.

8. Jacob E, Puntillo KA. A survey of nursing practice in the assessment and management of pain in children. Pediatr Nurs 1999;25(3):27886.
9. Vincent $\mathrm{CVH}$, Denyes MJ. Relieving children's pain: nurses' abilities and analgesic administration practices. J Pediatr Nurs 2004;19(1):40-50.

10. Simons J, Roberson E. Poor communication and knowledge deficits: obstacles to effective management of children's postoperative pain. J Adv Nurs 2002;40(1):78-86.

11. Watt-Watson J, Stevens B, Garfinkel P, Streiner D, Gallop R. Relationship between nurses' pain knowledge and pain management outcomes for their postoperative cardiac patients. $\mathrm{J}$ Adv Nurs 2001;36(4):535-45.

12. Barbosa SMM, Guinsburg R. Dor de acordo com as faixas etárias pediátricas. In: Teixeira MJ, Braum JL Filho, Marquez JO, Yeng LT. Dor: contexto interdisciplinar. Curitiba (PR): Maio; 2003. p. 535-45.

13. Helgadóttir HL. Pain management practices in children after surgery. J Pediatr Nurs 2000;15(5):334-40.

14. Woodgate R, Kristjanson LJ. A young child's pain: how parents and nurses' take care'. Int Nurs Stud 1996;33(3):271-84.

15. Pimenta CAM, Koizumi MS, Ferreira MTC, Pimentel ILC. Dor: ocorrência e evolução no pós-operatório de cirurgia cardíaca e abdominal. Rev Paul Enferm 1992;11(1):3-10.

16. Lellan KM. Postoperative pain: strategy for improving patient experiences. J Adv Nurs 2004;46(2):179-85. 
17. Huth MM, Broome MG, Mussatto KA, Morgan SW. A study of the effectiveness of a pain management education booklet for parents of children having cardiac surgery. Pain Manage Nurs 2003;4(1):31-9.

18. Knoblauch SC, Wilson CJ, Suzan C. Clinical outcomes of educating nurses about pediatric pain management. Outcomes Manag Nurs Practice 1999;3(2):87-9.

19. Hamers JPH, Abu-Saad HH, Hout MAVD, Halfens RJG. Are children given insufficient pain-relieving medication postoperatively? J Adv Nurs 1998;27:37-44

20. Pölkki T, Vehviläinen-Julkunen K, Pietilä AM. Nonpharmacological methods in relieving children's postoperative pain: a survey on hospital nurses in Finland. J Adv Nurs 2001;34(4):483-92.

21. Pölkki T. Nurses' perceptions of parental guidance in pediatric surgical pain relief. Int J Nurs Studies 2002;39:319-27.
22. Simons J, Franck L, Roberson E. Parent involvement in children's pain care: views of parents and nurses. J Adv Nurs 2001;36(4):591-9.

23. Romsing J, Moller-Sonnergaard J, Hertel S, Rasmussen M. Postoperative pain in children: comparison between ratings of children and nurses. J Pain Symptom Manage 1996;11(1):42-6.

24. Ministério da Saúde (BR). Secretaria de Gestão do Trabalho e da Educação na Saúde. Departamento de Gestão da Educação na Saúde. Política de educação e desenvolvimento para o SUS: caminhos para a educação permanente em saúde: pólos de educação permanente em saúde. Brasília (DF): Ministério da Saúde; 2004.

25. Huth MM, Broome ME, Good M. Imagery reduces children's post-operative pain. Pain 2004;110:439-48. 